\title{
LA RICERCA QUALITATIVA E LE QUESTIONI ETICHE
}

\section{THE QUALITATIVE RESEARCH AND THE ETHICAL QUESTION}

\section{A PESQUISA QUALITATIVA E AS QUESTÕES ÉTICAS}

\begin{abstract}
Angela Ales Bello ${ }^{1}$
Abstract: In this paper I deal with the questions of the logic of the research referring myself to the formation of the members of the ethical commissions, that are connected with a Platform Brazil. This Platform was constituted in order to evaluate the research projects according to a scientific, philosophical and ethical point of view. On my opinion it is necessary a discussion on the importance of the qualitative research particularly for the human sciences and the health sciences. From a philosophical perspective it is important to examine the qualitative research in connection with philosophical anthropology. For this reason I examine the role of ethics and to explain it, I go as far as Edmund Husserl's phenomenology to understand the constitution of the human being in himself/herself and the relationship with others. Moreover Husserl together with Hedwig Conrad-Martius gives us the criteria of a qualitative research not only regarding human sciences, but also regarding physical sciences. Very important is the role of the intellectuals; they have a great responsibility in general and particularly in the universities communities because of the consequence of their decisions for all the people in a country.
\end{abstract}

Keywords: Platform Brazil; Ethics; Qualitative Research; Plataforma Brasil; Scientific knowledge.

Resumo: Neste artigo trato de questões da lógica da pesquisa e as articulo com a formação de membros de comissões éticas que, no Brasil, estão em sintonia com a Plataforma Brasil. Essa Plataforma tem por finalidade a avaliação dos projetos de pesquisa para garantir a aceitabilidade dos mesmos em termos científicos, filosóficos e éticos. Destaco que em termos científicos é necessária a discussão sobre a importância, de modo particular, da pesquisa qualitativa para as ciências humanas e para as da saúde; em termos filosóficos tornam-se relevantes reflexões a respeito da lógica da pesquisa qualitativa e da antropologia filosófica. Por isso me concentrei no papel da ética e para entendê-lo voltei à filosofia fenomenológica de Edmund Husserl, que nos permite examinar o ser humano em si mesmo e em relação aos outros e, junto com Hedwig Conrad -Martius, fornece-nos os critérios de uma pesquisa qualitativa. Aponto pectos filosóficos e éticos que me parecem fundamentais para a formação humana em geral e em específico para os intelectuais que assumem posições de responsabilidade nos confrontos da comunidade universitária e enfatizo que a sua atitude repercute sobre a população toda.

Palavras-chave: Plataforma Brasil; Ética; Conhecimento científico; Pesquisa qualitativa.

\section{Introduzione}

Per affrontare il rapporto fra la ricerca qualitativa e le questioni etiche, è opportuno iniziare da queste ultime e chiedersi che cosa significhi etica. Spesso si dà per scontato il senso di questa parola, ma credo che in un congresso, che vuole approfondire tale questione in una dimensione pubblica, sia opportuno fermarsi a riflettere sull'etica.

\footnotetext{
${ }^{1}$ Professoressa Emerita di Storia dela filosofia contemporânea presso la Pontificia Università Lateranense di Roma e Presidente del Centro Iraliano di Riceche Fenomenologiche e dell'International Society of Phenomenology of Religion, com Sede a Roma. E-mail: alesbello@tiscali.it
} 
Ritengo che sia opportuno risalire al significato della parola per cogliere i modi in cui è stata intesa nel corso del pensiero occidentale. In senso ampio la parola greca $\varepsilon \theta \imath \kappa \dot{\text { indica }}$ la dimensione dei costumi, al di là della valutazione positiva o negativa di essi. In senso più specifico, nella tradizione filosofica ha spesso assunto proprio il significato di valutazione della condotta umana in conformità a principi e regole, che stabiliscano se tale condotta sia "morale". D'altra parte, è necessario osservare che la parola "morale" deriva dall'etimo latino mos e si riferisce anch'essa al costume. Nel corso del tempo ambedue le parole, quella greca e quella latina, hanno assunto un significato specifico, per indicare la sfera del comportamento umano nelle sue valenze positive e negative, comportamento che, quindi, deve essere sottoposto ad indagine per mettere in evidenza $\mathrm{i}$ criteri che sono alla base delle scelte attuate o da attuare.

Le parole: etica e morale, entrano, allora, nel linguaggio della filosofia occidentale per indicare, nella loro coincidenza totale o parziale, un settore specifico di ricerca. Poiché la speculazione filosofica affronta un intreccio di problemi riguardanti la realtà, quest'ultima può essere analizzata in modo da tentare di coglierla in tutta la sua ampiezza, oppure soffermandosi su aspetti parziali. Nel primo caso, si delineano le questioni che vanno sotto il titolo di "metafisica", connesse con l'esigenza di rintracciare le ultime ragioni di tutta la realtà che si presenta all'essere umano. Quest'atteggiamento si manifesta, in particolare, con Platone e Aristotele come indagine delle cause fondamentali che giustificano tutte le dimensioni, visibili e invisibili, della realtà. Nel secondo caso, sono affrontati argomenti specifici, ad esempio, la natura, la storia, la scienza, la religione ed anche l'etica. Si parla, perciò, del campo delle filosofie "seconde", considerate da taluni legate alla filosofia "prima", oppure completamente separate da essa da parte di coloro che considerano illusorio ogni discorso "metafisico". Da un punto di vista storico, si osserva che la frammentazione del sapere in varie ricerche specifiche è un fenomeno piuttosto recente nel pensiero occidentale.

Tuttavia, anche rimanendo sul piano delle filosofie seconde, non si può fare a meno di riconoscere una connessione fra esse. Come parlare, infatti, di comportamento morale, quindi, di questioni etiche, se non si sa che cosa sia l'essere umano? Ritengo, infatti, che ci sia uno stretto legame fra antropologia filosofica ed etica, legame che deve essere indagato. Allora, è opportuno iniziare proprio dall'antropologia. 


\section{Che cosa è l'essere umano?}

Nella tradizione filosofica e religiosa di molte culture l'essere umano è indicato come formato dal corpo e dall'anima. Anche nella storia della filosofia occidentale, si trovano molte interpretazioni concernenti il corpo, inoltre, la nozione di corpo rimanda sempre in positivo o in negativo alla nozione di anima, quindi, la questione del corpo deve essere trattata all'interno di una visione antropologica globale. Ci si può chiedere quale sia l'origine di tale distinzione. A mio avviso, essa nasce dall'esperienza del cadavere e dalla costatazione del venir meno di un principio vitale che lascia un corpo inerte e abbandonato. Allora che cosa è questo principio vitale? In quale rapporto è con il corpo? Poiché tendiamo a definire il corpo come materiale, ci si può domandare se il soffio vitale che lo anima sia materiale o immateriale.

In Occidente la distinzione fra anima e corpo sembra emergere in Eraclito; tale distinzione è sostenuta da Platone fino a considerare le due componenti come provenienti da "mondi" diversi, teorizzando, quindi, un netto dualismo, si attenua nel suo discepolo Aristotele, il quale riflette sul principio vitale, distinguendone i gradi, legati, appunto, alla capacità di avere sensazioni e reazione agli stimoli e, infine, alle possibilità conoscitive intellettive. Leggendo Aristotele riguardo all'esperienza del cadavere, si osserva che esso non può più toccare, vedere, avere impulsi, ragionare, parlare. Ma che cosa, in effetti, manca? Manca la possibilità di compiere una serie di operazioni, che dobbiamo analizzare più da vicino, domandandoci se il corpo così abbandonato, sia ancora corpo.

Rimaniamo sul piano della nostra esperienza. Se osserviamo un'altra persona, le operazioni alle quali mi riferivo prima sono colte dall'esterno, ma ognuno di noi le sente anche come proprie. Ma perché diciamo "un'altra persona"? Che cosa vuol dire "persona" e che cosa vuol dire "altra"? Che cosa scopriamo di diverso fra l'esperienza che abbiamo di un'altra persona e l'esperienza di un oggetto fisico?

Queste sono alcune delle domande intorno alle quali si affatica l'indagine filosofico-fenomenologica. Potrebbero essere considerate come domande banali, perché ci sembra di sapere che cosa vuol dire "altra persona". E' vero che lo sappiamo, ma anche comprendiamo che dobbiamo andare più a fondo, che non possiamo accontentarci di usare parole che hanno una lunga storia senza cogliere il senso che esse indicano. E' bene, pertanto, mettere fra parentesi le nozioni già acquisite e cercare di nuovo; ciò che è messo fra parentesi sussiste, non è negato, ma non lo facciamo agire, perché vogliamo soltanto indagare ulteriormente, per saggiarne la validità. Facendo ciò, siamo entrati già nel 
metodo fenomenologico. Abbiamo esercitato l'epoché, o sospensione del giudizio, per scavare, ad esempio, sulla genesi dell'espressione "un'altra persona". Possiamo dire, in prima battuta, che essa si fonda, appunto, su una serie di operazioni che sono da noi vissute, di cui abbiamo coscienza.

Abbiamo già individuato due parole-chiave in questo cammino regressivo: operazioni vissute e coscienza. Ognuno di noi può centrare la propria attenzione su se stesso e costatare che vive le sue esperienze in prima persona, ma scopre che sono vissute anche da altri, quindi, sono caratterizzate da una struttura comune, universale. Se sto percependo, la percezione è vissuta come qualcosa che è mi appartiene, eppure non è solo mia, anche altri possono viverla in quanto percezione; inoltre, essa non può essere confusa con esperienze vissute diverse, ha una sua caratteristica essenziale, è percezione e non è ricordo, non è immaginazione e così via. Quindi, ha un suo senso ed è contemporaneamente particolare, in quanto mia, e universale, in quanto presente anche in altri.

Proseguiamo nell'analisi della percezione. Percepisco qualcosa di "esterno" un tavolo. Che cosa mi fa dire: percepisco? Ciò che è esterno e correlato con ciò che è interno. Quando percepisco il tavolo, vuol dire che lo vedo e lo tocco, la mia mano ha una sensazione tattile, sento che essa mi appartiene, i messaggi che mi manda sono da me recepiti, ad esempio, la durezza del tavolo; fino ad un certo punto ci sono io che sento il tavolo e, poi, sento qualcosa che non sono più io, qualcosa che non mi appartiene più, è, appunto, esterno. Ma non è solo la mia mano che mi manda messaggi, incontro come estranea la sedia sulla quale sono seduto, il pavimento sul quale cammino. Queste cose, che sono per me anche ostacoli, mi consentono di stabilire "confini", i miei confini, sono aiutato in ciò anche dalla vista che mi fa vedere questi ostacoli, ma il tatto nel processo che abbiamo descritto è privilegiato nel delineare il mio limite, ma il limite di che cosa? Io dico del mio corpo. Il corpo, allora, si coglie attraverso le mie sensazioni che lo costituiscono e attraverso una serie di percezioni: il corpo si coglie da dentro.

Questo è un primo importane risultato; lo sento attraverso il tatto e vedo di esso solo alcune parti anteriori, ma so che c'è una parte posteriore, che posso toccare, ha, quindi, un suo "volume" e da esso non posso uscire percettivamente, ma solo attraverso l'immaginazione. Esso rappresenta un punto zero di orientamento in quello che si presenta fuori di me come uno spazio.

Nella costituzione del mio corpo, cioè nel modo in cui esso mi si dà, ho seguito le indicazioni di Edmund Husserl, in particolare quelle contenute nel secondo volume 
delle Idee per una fenomenologia pura e una filosofia fenomenologica (HUSSERL, 2002), trascritte da Edith Stein che poi le riutilizza nelle sue ricerche, soprattutto in $L a$ struttura della persona umana (STEIN, 2013).

L'indagine sulla corporeità, della quale ho dato solo brevi indicazioni, è un punto di partenza di un complesso cammino di ricerca, ma rappresenta un avvio indispensabile: in primo luogo, perché in esso è implicata la percezione, cioè la chiave d'accesso a tutto il processo conoscitivo, in secondo luogo, perché una volta entrati nella corporeità, subito si scopre che essa vive, manda segnali, ai quali si risponde attraverso gli stati psichici, le emozioni, i sentimenti e così via. Siamo in quella sfera che Husserl definisce, largamente, hyletica, usando la parola greca nel tentativo di indicare una dimensione che non è stata esaminata in tutta la sua potenzialità e che è la base per formazioni di grado superiore come, appunto, la costituzione di oggetti fisici. Inoltre, tale sfera non riguarda solo il rapporto del corpo proprio con ciò che è esterno, ma comprende anche altri gruppi di sensazioni che sono definiti "sentimenti sensoriali", come il piacere, il dolore, la tensione e il rilassamento, il benessere e il malessere che sono alla base della vita dei sentimenti e delle valutazioni e costituiscono il materiale (hyle) con il quale si formano i sentimenti superiori come l'amore e l'odio e le valutazioni delle situazioni prima di prendere decisioni con la volontà.

Entriamo, in tal modo, nella dimensione psichica strettamente connessa con il corpo e successivamente nella dimensione spirituale, cioè intellettuale e volontaria, propriamente umana. Il corpo che abbiamo sentito vivere è, pertanto, un corpo vivente senziente, non un semplice Körper, ma un Leib. Körper, in realtà, è più che un cadavere (Leichnam), perché usando questo termine s'intende la fisicità in quanto astratta da quel complesso vivente senziente, psicofisico che è il Leib. Quest'ultimo è un organismo che ha il suo movimento da dentro, movimento che è sentito e vissuto da un soggetto umano con consapevolezza cioè attraverso la coscienza e per tale ragione è un soggetto umano.

Il soggetto umano, in quanto essere spirituale, ha la capacità di valutare e di decidere, questi sono i presupposti fondamentali del suo comportamento che definiamo etico o morale e la valutazione richiede la presenza di criteri di orientamento. Da dove si traggono tali criteri? Vorrei fare riferimento a Edmund Husserl e alla sua insistenza sulle questioni etico-religiose, nel senso che in ogni cultura i comportamenti sono prevalentemente dettati in conformità con le credenze religiose. Nella cultura occidentale la tradizione religiosa dominante, come sappiamo, è quella ebraico-cristiana, la quale, anche se non sempre accettata e seguita in particolare in epoca più recente, ha lasciato le 
sue tracce nell'interpretazione dell'essere umano e le sue indicazioni nella convivenza sociale. Si tratta della valorizzazione dell'umano, il quale, perché figlio di Dio dal punto di vista religioso, ha una sua dignità che richiede rispetto. Quindi, i criteri che consideriamo positivi dal punto di vista etico sono il rispetto della persona umana; tutto ciò che promuove il bene sia in senso fisico sia in senso spirituale è considerato come un "valore".

L'etica presuppone la dimensione intersoggettiva nel senso che riguarda il comportamento fra gli esseri umani; infatti, come si è detto, non viviamo soli e il rapporto con gli "altri” è fondamentale.

\section{Il senso della ricerca}

L'essere umano è capace di fare molte cose, può dedicarsi a molte attività; per solito le distinguiamo in attività manuali e intellettuali. Se riflettiamo su questa distinzione, dobbiamo superare un pregiudizio secondo il quale le attività manuali escludono completamente la funzione dell'intelletto, cosa non vera perché qualsiasi lavoro ha bisogno di conoscenze, di regole e di capacità di invenzione di fronte a situazioni nuove. Il fare, nel senso del produrre pratico, non solo introduce cose nuove nell'ambito intersoggettivo, ma le finalizza a scopi precisi. E l'attività puramente intellettuale in che misura si distingue da quella "manuale"? Indubbiamente ha bisogno di conoscenze "teoriche" in grado maggiore, lavora per approfondirle e per allargare gli orizzonti del sapere nei vari ambiti e tale lavoro e quello che definiamo il lavoro di "ricerca". Che cosa si cerca? Creazione di strumenti funzionali, creazione artistica, creazione filosofica. Qual è la differenza? Sta nel coinvolgimento di elementi fisici. Gli strumenti hanno bisogno di "materie prime", di oggetti della natura, ma anche gli artisti hanno bisogno della natura, si pensi ai pittori, agli scultori, ai musicisti e i poeti. Questi ultimi si avvicinano ai filosofi perché apparentemente non hanno bisogno di oggetti naturali, ma ciò è vero fino ad un certo punto e ciò è dimostrato dai testi del poeta greco Omero o da quelli che consideriamo i primi filosofi sempre in lingua greca: se non ci fossero i loro scritti non potremmo sapere ciò che hanno detto, allora serve anche la pietra, la pergamena, lo scalpello o l'inchiostro per tramandare il sapere, nei nostri giorni indispensabili sono gli strumenti elettronici, ad esempio.

Allora, si può comprendere che qualcuno dica che è la "quantità" di materia o di materiali che fa la differenza, che la misura è alla base della costruzione degli strumenti. 
E ciò è vero! Ma qual è la finalità della ricerca? Non la quantità, ma la qualità delle "cosa" ricercata, cioè, che sia funzionante, che sia bella in senso estetico, che esprima della verità in senso poetico, che colga il senso delle cose in filosofia. E lo studio della natura in quanto tale è quantitativo o qualitativo? Si apre qui una questione teorica che ha una grande incidenza pratica, perché la conoscenza della natura consente le trasformazioni che riguardano la tecnica e, quindi, la costruzione e gli usi degli strumenti che consentono di trasformare anche la natura. Ciò è stato possibile in gran parte attraverso un procedimento quantitativo, quella che Husserl definisce la gabbia concettuale di tipo matematico che Galilei ha messo sulla natura.

\section{Ricerca qualitativa e ricerca quantitativa}

Soffermiamoci, allora, su questa critica husserliana contenuta nella sua ultima opera La crisi delle scienze europee e la fenomenologia trascendentale (HUSSERL, $1987)^{2}$. La scienza fisica pretende di conoscere la natura "misurandola", ma la natura, intesa come universo matematico rimane sempre una, ma la tematizzazione è "indiretta", infatti, una tematizzazione diretta è impossibile, perché “... le qualità specificamente sensibili dei corpi" ${ }^{3}$ fuggono alla misurazione "esatta", quella che vorremmo raggiungere come un ideale. Tali qualità sensibili mostrano che i corpi fisici sono dei "plena", qualcosa di pieno, che sfugge sempre alla pura riduzione in forme matematiche. I plena sono forme in senso qualitativo.

La posizione di Husserl trova una conferma nelle indagini di Hedwig ConradMartius, anch'ella sua discepola, la quale introduce la nozione di trans-fisico, intendendo che i corpi fisici hanno sempre forme di tipo qualitativo che li caratterizzano. Ella, interessata alle scienze biologiche, esamina in particolare l'organismo vivente e sostiene che è caratterizzato da un'entelechia, cioè da una modalità essenziale tipica che lo costruisce e lo rigenera. Si tratta della ripresa dell'interpretazione di Aristotele che aveva messo in evidenza la "forma", quindi, la qualità degli esseri viventi. In realtà, esistono due entelechie: una che presiede alla formazione e allo sviluppo dell'organismo che è definita, appunto, trans-fisica, perché supera la corporeità intesa in modo materiale, e l'altra che caratterizza l'identità individuale tipica, la vera e propria essenza metafisica. Questa interpretazione si trova in libro intitolato L'autocostituzione della natura.

\footnotetext{
${ }^{2} \mathrm{Si}$ veda in particolare il $\S 9$, La ma tematizzazione galileiana della natura.

${ }^{3}$ Ivi, §9, c, p.63.
} 
Entelechie ed energie (CONRAD-MARTIUS, 1961) ${ }^{4}$. Ciò non esclude le indagini di tipo fisiologico o l'utilizzazione di strumenti diagnostici, ma sappiamo come sia difficile una standardizzazione nell'ambito della medicina perché ogni organismo ha sue caratteristiche specifiche e reagisce in modo personale alle medicine che sono somministrate, ad esempio.

Ho introdotto le due proposte teoretiche di Husserl e della Conrad-Martius per mostrare che la ricerca quantitativa è possibile, ma non descrive fino in fondo il senso della realtà, neppure di quella naturale, ciò significa che la scienza fisica è insufficiente per conoscerla, è necessaria anche una filosofia della natura.

$\mathrm{Ci}$ si potrebbe chiedere quale sia il legame tra le questioni conoscitive nei vari campi del sapere e l'etica, sembrerebbe due mondi diversi.

\section{Ricerca qualitativa ed etica}

Preliminarmente è opportuno osservare che se la natura ha bisogno di una ricerca qualitativa, è chiaro che l'essere umano non può essere letto in modo quantitativo e questo per una duplice ragione: perché appartiene alla natura e perché eccede rispetto ad essa. L'eccedenza è rappresentata proprio dall'attività spirituale, che è alla base dell'organizzazione del sapere e che dirige il comportamento umano. A questo proposito mi sembra interessante il rapporto fra la psiche e lo spirito, perché è in questo rapporto che si gioca il comportamento positivo o negativo dell'essere umano.

La psiche è il luogo degli istinti, delle reazioni di attrazione o repulsione, quindi, delle pulsioni, delle emozioni, che devono essere valutate per stabilire se devono essere seguite o no. Abbiamo visto che importanti sono i criteri di valutazione, ma non è sempre facile procedere a tale valutazione e staccarsi dalle pressioni psichiche. Ciò non vuol dire che esse siano sempre negative, anzi ci sono alcuni moti psichici molto positivi, di apertura verso gli altri, si tratta di vagliare nelle singole situazioni e decidere che cosa fare. La decisione può seguire la valutazione, ma in alcuni casi segue direttamente ciò che la psiche suggerisce e, quindi, si sottomette ad essa. Sperimentiamo ciò in ogni momento della nostra vita e ciò contribuisce anche alla "fatica" del vivere. La responsabilità consiste nel valutare secondo criteri validi e operare in conformità con essi.

\footnotetext{
${ }^{4}$ Ho analizzato la posizione di Conrad-Martius sulla natura nel mio libro Fenomenologia dell'essere umano. Lineamenti di una filosofia al femminile, Città Nuova, Roma, 1992, tradotto in portoghese Fenomenologia do ser humano. tr. di A. Angonese, EDUSC, Bauru - Brasile, 2000.
} 


\section{L'etica alla prova dei fatti}

$\mathrm{Ci}$ si domandava sopra quale fosse il legame fra la ricerca nei vari campi del sapere e l'etica, perché sembrerebbe che quando ricerchiamo siamo soli. Ma tale solitudine è solo apparente, in realtà, siamo sempre più o meno direttamente in contatto con gli altri. Esiste una "onestà intellettuale" che deve essere esercitata. In primo luogo l'onestà è connessa al non appropriarsi delle idee altrui senza citare la fonte, al non ricercare solo per avere successo e non per vero interesse, al non avere dei pregiudizi e cercare di avvalorarli con risultati che non sono validi. Ci sono poi anche altri aspetti, ad esempio, le manipolazioni per aumentare i guadagni senza tener conto delle conseguenze delle azioni, oppure per fini di consenso politico. Dipende certamente dagli ambiti d'indagine. Quali sono i criteri che bisogna osservare affinché una ricerca possa essere definita conforme all'onestà intellettuale? Direi che il criterio fondamentale è quello del "rispetto" di se stessi, degli altri, della natura e dei prodotti artificiali.

Vorrei citare una conferenza di Edith Stein che bene si connette alle riflessioni che stiamo facendo, s'intitola L'intelletto e gli intellettuali (STEIN, 2015) e tratta dell'incidenza che la ricerca intellettuale può avere sul popolo. Mi sembra che in ultima analisi si sottolinei la responsabilità degli intellettuali riguardo alla società e alla politica. Ella insiste sulla formazione "morale" dell'intellettuale che non deve staccarsi dagli altri, ma deve essere al loro servizio, seguendo criteri che definisce "sapienziali". La sapienza si base sulle conoscenze specifiche, ma anche sull'atteggiamento morale, che s'ispira alla dimensione religiosa. In ultima analisi che cosa è "bene"?

Si può applicare tutto ciò ad alcuni casi specifici. Nel congresso che si è tenuto in settembre 2021 il sesto della SIPEQ si è discusso sulla logica della ricerca qualitativa, ma anche delle questioni legate alla formazione dei comitati etici che devono lavorare in sintonia con la piattaforma Brasil finalizzata alla valutazione dei progetti di ricerca per garantirne l'accettabilità in termini scientifici, filosofici ed etici.

L'espressione: "In termini scientifici" significa che è necessaria la discussione sull'importanza della ricerca qualitativa, in particolare, per le scienze umane e quelle della salute, "in termini filosofici" rimanda alla riflessione sulla logica della ricerca qualitativa, ma anche in modo più ampio alla questione dell'antropologia filosofica che diventa una base necessaria non solo per la logica, ma anche per l'etica. A questo scopo ho cercato di indagare sugli aspetti filosofici ed etici che mi sembrano fondamentali per la formazione umana in generale e in modo specifico per gli intellettuali che svolgono mansioni di 
"responsabilità" nei confronti della comunità universitaria, perché, come abbiamo già detto, il loro atteggiamento ha ripercussioni sulla popolazione. La loro formazione dovrebbe essere completa dal punto di vista delle conoscenze del loro campo di ricerca e questo ha una relazione profonda con la morale, perché è un dovere prepararsi in modo valido per svolgere bene il proprio lavoro e cercare con disponibilità quali sono le condizioni migliori perché una ricerca sia efficace. Si è mostrato che la ricerca qualitativa è quella che consente di conoscere meglio se stessi, gli altri esseri umani e anche la natura. Allora chi partecipa ad un comitato etico ed ha la responsabilità di esaminare progetti, deve avere chiaro il significato della ricerca, deve essere disponibile ed anche responsabile, non deve farsi influenzare da motivi estrinseci alla ricerca stessa, deve esercitare la giustizia e valutare senza pregiudizi.

La preparazione personale e lo sforzo interiore di perfezionare se stessi potranno dare frutti importanti nella comunità scientifica. Le questioni etiche hanno incidenza sulla vita delle Università, ma anche sulla vita del popolo.

\section{Riferimenti}

HUSSERL, E. Idee per una fenomenologia pura e una filosofia fenomenologica. Tr. it a cura di Vincenzo Costa. Einaudi: Torino 2002.

STEIN, E. La struttura della persona umana. Corso di antropologia filosofica. Tr. it. di M.D’Ambra, Revisione di A. M. Pezzella e M. Paolinelli, a cura di Angela Ales Bello e Marco Paolinelli. Città Nuova: Edizioni OCD Roma, 2013.

HUSSERL, E. La crisi delle scienze europee e la fenomenologia trascendentale. Tr.it. di E, Fillipini, Il, Saggiatore. Milano: [s.n.], 1987.

CONRAD- MARTIUS, H. Der Selbstaufbau der Natura. Entelechien und Energien. München: Kösel - Verlag, 1961.

STEIN, E. Gli intellettuali. Tr. it. di A. Togni. Introduzione di A. Ales Bello. Roma: Castelvecchi, 2015.

Convite feito em: 10 de maio de 2021

Revisão feita em: 25 de novembro de 2021 